J. Geophys. Res., 107 (D19), 4395, doi:10.1029/2001JD001029, 2002

\title{
Temperature Profile Determination from Microwave Oxygen Emissions in Limb Sounding Geometry
}

\author{
Axel von Engeln ${ }^{1,2}$, Stefan Bühler ${ }^{1}$
}

\begin{abstract}
We present a theoretical investigation of the temperature profile retrieval capabilities of oxygen emission lines in the microwave. The main focus is on two strong lines, both allowing temperature retrieval throughout the mesosphere. One is within the oxygen cluster at $61.15 \mathrm{GHz}$, the other one is isolated at $118.75 \mathrm{GHz}$. A thorough comparison of these two lines is presented. Several instrumental parameters such as system noise temperature, antenna beam width, filter width, and coverage of the line are assessed, as well as the possible impact of an error in the spectroscopic parameters. The instrumental setup follows roughly the specifications for the MASTER instrument, serving as a basis for a modern passive microwave instrument. The instrumental parameters have also been varied in order to allow comparisons with two instruments that use the $118.75 \mathrm{GHz}$ line for temperature profile determination, the ODIN-SMR and the EOS-MLS. Simultaneous retrieval of temperature and pointing bias is performed with the Optimal Estimation Method. We find temperature retrieval errors of $<5 \mathrm{~K}$ in the mesosphere, and at sub-Kelvin level in the lower stratosphere. Good knowledge of spectroscopic parameters is required for accurate retrievals. The simultaneous retrieval of a pointing bias can reduce the impact of spectroscopic parameter errors on the temperature retrieval.
\end{abstract}

\section{Introduction}

Atmospheric temperature is the single most important parameter when dealing with the atmosphere in areas such as meteorology, photochemistry, or climatology. Atmospheric temperatures can be retrieved remotely by measuring the thermal emission at an appropriate wavelength. The retrieval problem is greatly simplifi ed if one assumes that local thermodynamic equilibrium (LTE) is fulfi lled in the atmospheric layers from which the radiation originates. Since the existence of LTE depends heavily on pressure and wavelength, it is important to select the observing wavelength accordingly. For observations in the thermal infrared range (near $10 \mu \mathrm{m}$ wavelength), LTE generally holds to $\sim 50 \mathrm{~km}$ altitude. For the microwave range, the LTE is valid up to $100 \mathrm{~km}$.

Thermal emissions of molecular oxygen lines are particu-

\footnotetext{
${ }^{1}$ Institute of Environmental Physics, University of Bremen, Bremen, Germany

${ }^{2}$ George Mason University Fellow at Naval Research Laboratory, Washington, D.C., USA
}

larly useful for the determination of the atmospheric temperature profi le, owing to the known and constant VMR profi le. At microwave frequencies there are a variety of observable oxygen lines between $50 \mathrm{GHz}$ and $1 \mathrm{THz}$. The lines at the lower frequencies are the result of spin flip transitions at different rotational levels [van Vleck, 1947]. Almost all of these lines occur in a cluster around $60 \mathrm{GHz}$, with the exception of one line at $118 \mathrm{GHz}$. The spin $S$ results from two unpaired electrons leading to $S=1$ for the oxygen molecule. The orbital angular momentum is zero, and the spin angular momentum is coupled to the rotational angular momentum through a weak magnetic fi eld arising from the molecular rotation. This coupling can be approximated by a Hund's case b [Gordy and Cook, 1970; Mizushima, 1975]. The vector addition of $S$ and the rotational quantum number $N$ leads to a total of three levels of the total angular momentum $J$ per rotational level. Two different transitions are of importance in the microwave: from the $J=N$ to the $J=N+1$ level (designated as $N+$ lines) and between the $J=N$ and the $J=N-1$ level (designated as $N-$ lines). Only odd $N$ values are allowed because of the symmetry requirements. 
Each $J$ level will be affected by the Zeeman effect and will split up into $(2 J+1)$ sublevels when a magnetic fi eld is applied.

Accurate modeling of the Zeeman effect requires a polarized radiative transfer calculation through the atmosphere. Both the position of the sensor and the tangent point have to be taken into account with respect to the strength and direction of the magnetic fi eld.

Passive microwave instruments in a limb sounding mode can take advantage of the strong oxygen emissions to obtain high vertical resolution temperature profi les. The line spectrum around $60 \mathrm{GHz}$ and $118 \mathrm{GHz}$ is particularly useful, owing to the strong intensity of these lines, allowing stratospheric and mesospheric temperature determination. These microwave oxygen lines have been observed in limb sounding geometry in the past by the MLS [Barath et al., 1993] and the MAS [Croskey et al., 1992] instruments, both focusing on strong lines within the oxygen cluster around $60 \mathrm{GHz}$. Atmospheric temperature profi le retrievals from these observations have been presented by Fishbein et al. [1996] for the MLS instrument, and by Wehr et al. [1998] and von Engeln et al. [1998] for the MAS instrument.

The MLS temperature retrieval is performed on a pressure grid and allows single temperature profi le determination with an accuracy of about $1.5 \mathrm{~K}$ at $22 \mathrm{hPa}(\approx 25 \mathrm{~km})$ and about $3 \mathrm{~K}$ at $0.46 \mathrm{hPa}(\approx 57 \mathrm{~km})$ [Fishbein et al., 1996]. The MAS retrieval is performed on an altitude grid and single profi le retrievals are possible with accuracies of about $3 \mathrm{~K}$ at $20 \mathrm{~km}$, about $2 \mathrm{~K}$ at $50 \mathrm{~km}$, and about $6 \mathrm{~K}$ at $90 \mathrm{~km}$ [von Engeln et al., 1998].

Newer instruments such as the ODIN-SMR [Eriksson, 1999], which was launched in February 2001, and the EOSMLS instrument [Waters, 1999], which is part of NASA's EOS-Aura mission, focus on the single oxygen line at $118 \mathrm{GHz}$.

A new approach to determine temperature profi les from microwave limb measurements of several emission lines has been presented by Verdes et al. [2000]. This approach can be used to obtain temperature information from other molecules that have several strong spectral features, such as ozone. In general, this restricts the accessible altitude region to the stratosphere where these spectral features are strong.

In this paper we study the temperature retrieval capabilities of microwave oxygen emissions lines in limb sounding geometry for stratospheric and mesospheric observations. We focus on two strong lines, the strongest line within the oxygen cluster at $61.15 \mathrm{GHz}$, and an isolated line at $118.75 \mathrm{GHz}$. General retrieval capabilities for these and several other lines, as given in Table 1, are investigated. The lines are identifi ed by their rotational quantum number as mentioned above. The maximum intensity found in all spec- tral channels observed at a tangent altitude of $90 \mathrm{~km}$ is included as well.

Sections 2 and 3 describe the underlying forward and retrieval model. Section 4 presents the retrieval results, separated into general retrieval capabilities, and a more detailed investigation into the two main lines. Firstly, the impact of the instrumental parameters is shown, and secondly, the impact of spectroscopic errors. Finally, a conclusion summarizes the fi ndings.

\section{Forward Model Setup}

The characteristics of the passive microwave instrument to be used as the basis for this study have been taken from the specifi cations of the MASTER instrument [Reburn et al., 2000]. This instrument is part of the ACECHEM (Atmospheric Composition Explorer for Chemistry and Climate Interaction) mission, a possible European Space Agency Earth Explorer candidate. The MASTER instrument characteristics have been derived in close cooperation with research and development to assure a realistic setup. The instrument does not observe the discussed oxygen lines, but the instrumental characteristics serve as a basis for a modern passive microwave instrument. We shall assume limb sounding measurements from an orbit of $820 \mathrm{~km}$ with a $2 \mathrm{~m}$ antenna, integrating for $0.2 \mathrm{~s}$ per spectrum at each tangent altitude. The integration time within this study was slightly lowered in comparison to the MASTER specifi cations $(0.3 \mathrm{~s})$, because the MASTER scan is limited to the lower atmosphere, and the integration time has been reduced in order to get a similar number of profi les per orbit.

In this study we assume a slightly higher orbit than the ODIN satellite (about $600 \mathrm{~km}$ [Eriksson, 1999]), or the EOSAura orbit (about $700 \mathrm{~km}$ [Waters, 1999]). The atmospheric scenario employed was a mid-latitude summer one, with the instrument position at $70^{\circ} \mathrm{N}$ and $10^{\circ} \mathrm{E}$, the tangent point at $43^{\circ} \mathrm{N}$ and $10^{\circ} \mathrm{E}$, the orbital inclination at $90^{\circ}$, and observations in the orbit plane. The magnetic field at the tangent point is about $45 \mu \mathrm{T}$ and the angle between the magnetic fi eld and observation direction is about $60^{\circ}$ [Barraclough, 1986]. Uncertainties in the model of the Earth's magnetic fi eld are suffi ciently small and effects on the instrumental capability can be disregarded.

The instrument measures the spectral power y over a range of wavelengths. We can model this spectrum using the notation: $\mathbf{y}=F(\mathbf{x}, \mathbf{b})$, where $F$ denotes the Forward model, $\mathbf{x}$ the unknown (retrieved) variables, e.g., VMR profi les and instrument parameters, and $\mathbf{b}$ the model parameters, e.g., fi xed VMR profi les or spectroscopic data, which are not retrieved, but introduce systematic errors.

For this study we shall use a forward model that has been 
Table 1. Investigated oxygen lines.

\begin{tabular}{ccc}
\hline Line & Frequency $[\mathrm{GHz}]$ & Intensity at $90 \mathrm{~km}$ \\
\hline $9+$ & 61.15 & 138.9 \\
$1-$ & 118.75 & 145.2 \\
$17+$ & 63.57 & 58.7 \\
$19+$ & 64.13 & 33.0 \\
$21+$ & 64.68 & 16.0 \\
$25+$ & 65.76 & 3.4 \\
\hline
\end{tabular}

previously used for temperature retrieval from MAS observations [von Engeln et al., 1998]. This forward model is a polarized line-by-line radiative transfer model, which includes the Earth's magnetic fi eld and considers contributions from all oxygen, water vapor, and ozone lines within the investigated frequency range. We assume an instrumental scan range from $0 \mathrm{~km}$ to $110 \mathrm{~km}$, in steps of $1 \mathrm{~km}$. The nominal observation scenario assumes a $1 \mathrm{GHz}$ wide band centered on the line of interest. Coverage of the line is provided by $3 \mathrm{MHz}$ fi lters for the outer regime of the line, and $0.1 \mathrm{MHz}$ for the inner regime $( \pm 4 \mathrm{MHz}$ around the line center). The instrument is assumed to observe one sideband only (Single Sideband SSB), with a system noise temperature of $1000 \mathrm{~K}$. It observes a linear component of the emitted polarized radiation, where the orientation is along the scan direction. The angle between the projected magnetic fi eld in the plane perpendicular to the integration path and the scan direction in this plane is assumed to be about $90^{\circ}$ at the tangent point.

The brightness temperature spectra of the $61.15 \mathrm{GHz}$ and the $118.75 \mathrm{GHz}$ line calculated with the forward model described above is presented in Figure 1 for different tangent altitudes.

\section{Retrieval Model}

The inverse model $I$ calculates the most likely solution $\hat{\mathbf{x}}$ of the real atmospheric state $\mathbf{x}$. An iterative approach is necessary because the retrieval of temperature from microwave measurements is in general non-linear. The retrieval algorithm used here is based on the Optimal Estimation Method (OEM), for a more detailed description please refer to Rodgers [2000]. It uses a priori knowledge on the state of the atmosphere to stabilize the solution.

The iterative formula to calculate $\hat{\mathbf{x}}$ for the iteration $n+1$ is given as:

$$
\hat{\mathbf{x}}_{n+1}=\mathbf{x}_{0}+\mathbf{G}_{n}\left[\left(\mathbf{y}-\mathbf{y}_{n}\right)-\mathbf{K}_{n}\left(\mathbf{x}_{0}-\hat{\mathbf{x}}_{n}\right)\right]
$$

where $\mathbf{x}_{0}$ is the a priori vector from which the iteration starts,
$\mathbf{G}_{n}$ the $n$-th iteration of the gain matrix, $\mathbf{y}_{n}$ the forward model output, and $\mathbf{K}_{n}$ the weighting function matrix.

The Jacobian matrices $\mathbf{K}_{n}$ and $\mathbf{G}_{n}$ are defi ned as:

$$
\left.\left.\mathbf{K}_{n} \equiv \frac{\partial F}{\partial \mathbf{x}}\right|_{\mathbf{x}=\mathbf{x}_{n}} \quad \mathbf{G}_{n} \equiv \frac{\partial I}{\partial \mathbf{y}}\right|_{\mathbf{y}=F\left(\mathbf{x}_{n}\right)}
$$

For this study the Jacobian matrix $\mathbf{K}_{n}$ is calculated by perturbing the corresponding retrieval parameter $\mathbf{x}$ of $F(\mathbf{x}, \mathbf{b})$. The matrix $\mathbf{G}_{n}$ can be calculated from:

$$
\mathbf{G}_{n}=\left(\mathbf{S}_{0}^{-1}+\mathbf{K}_{n}^{T} \mathbf{S}_{y}^{-1} \mathbf{K}_{n}\right)^{-1} \mathbf{K}_{n}^{T} \mathbf{S}_{y}^{-1}
$$

with the a priori covariance matrix $\mathbf{S}_{0}$, the error covariance matrix of the measured spectrum $\mathbf{S}_{y}$, and $\mathbf{K}_{n}^{T}$ denotes the transpose matrix of $\mathbf{K}_{n}$.

The OEM allows the calculation of two statistical errors, each of which contributes to the total error of the retrieval. Generally one can write the total retrieval error covariance matrix $\mathbf{S}$ :

$$
\mathbf{S}=\mathbf{S}_{S}+\mathbf{S}_{M}
$$

where $\mathbf{S}_{S}$ is the smoothing error covariance matrix, and $\mathbf{S}_{M}$ is the measurement error covariance matrix. Furthermore, one defi nes the averaging kernel matrix $\mathbf{A}$ as:

$$
\mathbf{A}=\mathbf{G K}
$$

Making use of this notation the individual error matrices can be calculated as:

$$
\begin{aligned}
\mathbf{S}_{S} & =(\mathbf{A}-\mathbf{I}) \mathbf{S}_{0}(\mathbf{A}-\mathbf{I})^{T} \\
\mathbf{S}_{M} & =\mathbf{G S}_{y} \mathbf{G}^{T}
\end{aligned}
$$

where $\mathbf{I}$ denotes the identity matrix. It is assumed within this study that $\mathbf{S}_{0}$ in Eq. 6 is identical to the a priori knowledge $\mathbf{S}_{0}$ in Eq. 3.

The smoothing error represents the error in those portions of profile space where the observing system is insensitive. 

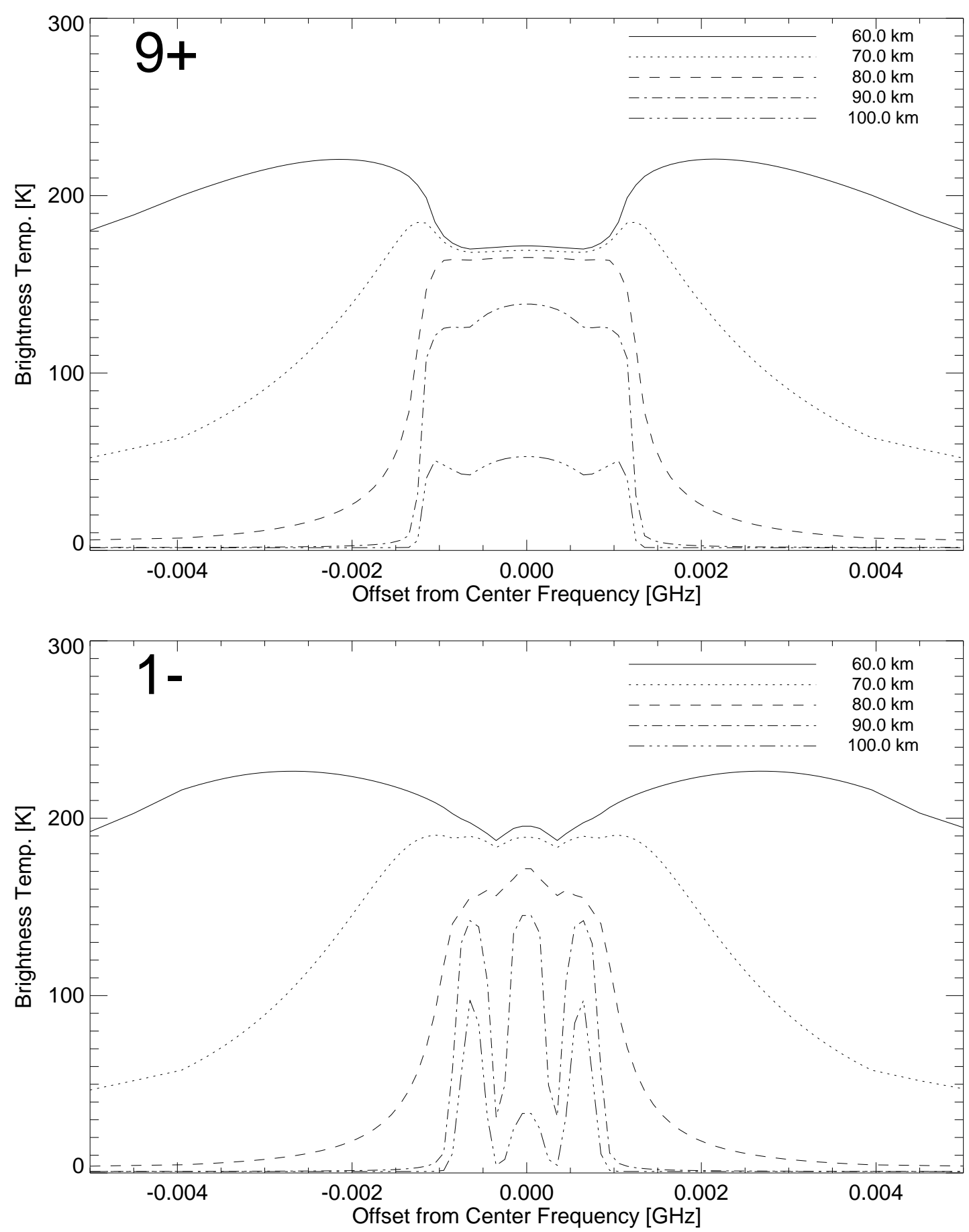

Figure 1. Spectra of the $61.15 \mathrm{GHz}$ (top) and the $118.75 \mathrm{GHz}$ line (bottom) for different tangent altitudes. 
This might be values of the profi le below the lowest scan tangent altitude for a limb sounder, or small scale variations that cannot be detected due to the limited altitude resolution. The measurement error is due to noise in the measured spectrum that has propagated into the retrieval.

A third error is associated with errors in forward model parameters that are not retrieved but assumed to be known:

$$
\mathbf{S}_{F}=\mathbf{G K}_{b} \mathbf{S}_{b}\left(\mathbf{G} \mathbf{K}_{b}\right)^{T}
$$

where $\mathbf{S}_{F}$ is called model parameter error covariance matrix and $\mathbf{S}_{b}$ is the corresponding error covariance matrix of the spectroscopic parameters. The matrix $\mathbf{K}_{b}$ is calculated similar to $\mathbf{K}$ by perturbing the corresponding model parameter $\mathbf{b}$ of $F(\mathbf{x}, \mathbf{b})$. The total error budget of a retrieval would consist of the sum of $\mathbf{S}$ and $\mathbf{S}_{F}$. However, we discuss these errors separately in this study to get a better picture of the retrieval process.

We shall assume that the spectrum error covariance and the a priori error covariance are statistical in nature (i.e., the measurement itself and the a priori profi le have no systematic bias). This assumption is not necessarily fulfi lled for the model parameter error. If the associated model parameter is for example part of the spectroscopy (e.g., a pressure broadening coeffi cient), the resulting error will be systematic.

The averaging kernel matrix $\mathbf{A}$ as given in Eq. 5 is a very useful tool. The averaging kernel functions (defi ned as the rows of the averaging kernel matrix) at a certain altitude shows how the retrieval at this altitude is sensitive to changes at other altitudes. For a perfect measurement system (Measurement system here means everything that is described by the forward model, not just the instrument) it would be unity at that altitude and zero everywhere else. In practice the averaging kernel function will have a fi nite width. The averaging kernel gives information about the resolution of the retrieval, and the influence of the a priori information. At altitudes where the sum of the averaging kernels is near unity the retrieval value of $\hat{\mathbf{x}}$ will be primarily determined by the measurement, while at altitudes where the sum is near zero it will be primarily determined by the a priori.

\subsection{Retrieval Setup}

The $\hat{\mathbf{x}}$ vector describes in this investigation the temperature profi le between $0 \mathrm{~km}$ and $100 \mathrm{~km}$. Additionally, a pointing bias is retrieved (see section on pointing bias retrieval). The $\mathbf{S}_{0}$ matrix is generated with a $10 \mathrm{~K}$ a priori uncertainty on temperature, and a pointing bias uncertainty of about $1 \mathrm{~km}$. The matrix $\mathbf{S}_{b}$ is generated with a $5 \%$ uncertainty on the investigated spectroscopic parameters (line intensity, line width, overlap) and $0.01 \mathrm{MHz}$ on the line center frequency. The measurement covariance matrix $\mathbf{S}_{y}$ is generated from the measurement error, which can be calculated according to the radiometer equation as:

$$
\Delta T=\frac{T_{s y s}}{\sqrt{W \tau}}
$$

where $\Delta T$ is the noise per filter channel, $T_{s y s}$ denotes the system noise temperature, $W$ the filter width, and $\tau$ the integration time. No correlations were assumed for $\mathbf{S}_{0}, \mathbf{S}_{b}$, or $\mathbf{S}_{y}$, i.e. the matrices are diagonal.

A Numerical Weather Prediction (NWP) model forecast calculation can provide an a prior temperature profi le. The forecast uncertainty of a NWP model increases from $2 \mathrm{~K}$ at the ground to about $10 \mathrm{~K}$ at $60 \mathrm{~km}$ [Palmer et al., 2000]. Thus a constant a priori uncertainty of $10 \mathrm{~K}$ for all altitudes is a conservative estimate of our current knowledge of the atmosphere up to $60 \mathrm{~km}$. Current knowledge of temperatures above $60 \mathrm{~km}$ is limited, we use a $10 \mathrm{~K}$ a priori uncertainty as an ad hoc assumption. Overall, a uniform a priori uncertainty has the advantage that the sensitivity to measurement errors at different retrieval altitudes is more easily comparable. The a priori temperature profi le has to be chosen very carefully for a retrieval from real data, in order to avoid the introduction of a bias.

We assumed that the $5 \%$ spectroscopic parameter uncertainty can be achieved by dedicated laboratory measurements [Bauer et al., 1998], while an error of $0.01 \mathrm{MHz}$ is given for the line center frequency in the online version of the JPL catalog [Pickett and Poynter, 1985].

\subsection{Pointing Bias Retrieval}

In addition to temperature, a pointing bias is retrieved in order to correct tangent altitude pointing information from the attitude and orbit control system (AOCS) of the spacecraft. Pointing bias information comes from two different sources: (1) The measurement itself provides pressure and thus pointing information, mainly due to the pressure broadening of the lines. (2) The spacecrafts AOCS provides information about the geometric tangent altitudes (which differ slightly from the observed tangent altitudes due to refraction).

The tangent altitude information for the MASTER instrument from the AOCS is expected to be around $1 \mathrm{~km}$ (J. Langen, Atmospheric Unit, ESA-ESTEC, personal communication, 2001). A much more accurate estimate of the pointing can be obtained by comparing the retrieved pressure at one of the grid points (we use the $32 \mathrm{~km}$ grid point, where this pressure is used as well to generate the pressure profi le by employing the hydrostatic equation) with an NWP model. The difference between the nominal altitude from the AOCS and the altitude derived from the NWP model is then used 
to derive a pointing bias. Because the relative pointing provided by the AOCS is likely to be quite accurate, we can use the AOCS to provide pointing information for all other altitudes.

The actual retrieval capabilities regarding temperature and pointing bias are almost independent of the chosen a priori error on the initial pressure value, since enough information is provided by the measurement itself. The chosen $15 \%$ uncertainty corresponds to a pointing bias error of about $1 \mathrm{~km}$.

The impact of refraction on the observed tangent altitude is not considered in this study but must be considered for retrieval from real measurements. This simplification was made for computational reasons and does not limit the outcome of this study since this effect can be very accurately modeled. The change for a tangent altitude observation at $20 \mathrm{~km}$ is about $100 \mathrm{~m}$ [Eriksson, 1999] and decreases exponentially with altitude. The effect is almost independent of frequency for the considered frequency range (see Liebe and Hufford [1989] for more information).

\section{Retrieval Results}

General retrieval capabilities of several oxygen lines are investigated, with a focus on the $61.15 \mathrm{GHz}$ and the $118.75 \mathrm{GHz}$ line. A detailed study of the possible vertical retrieval grid is performed fi rst. Applying the derived vertical retrieval grid, the weighting function matrix $\mathbf{K}$ is used to visualize which fi lters provide temperature information at a specifi $\mathrm{c}$ altitude. An assessment of the instrumental parameters is performed. In addition, the impact of spectroscopic errors on the retrieval capabilities is addressed. The impact of the magnetic fi eld on the retrieval accuracy has not been addressed here, please refer to von Engeln et al. [1998] for a more detailed description.

\subsection{Vertical Retrieval Grid}

Generally, a vertical retrieval grid is affected by 3 considerations: (1) The information provided by the measurement. (2) The required resolution for atmospheric modeling. (3) Computer resources.

Assuming that computer resources are given, one has to fi nd a compromise between (1) and (2). The measurement contains more information for lower tangent altitudes, due to the higher density of the atmosphere. Thus the highest possible resolution should be chosen, only limited by measurement noise. For modeling purposes it is desirable to have a high resolution in the mesosphere as well, but information is limited and a higher resolution leads to higher retrieval errors. We assumed as a threshold for a meaningful retrieval an improvement of the total retrieval error over the a priori uncertainty of about $50 \%$.

One way to determine the vertical retrieval resolution is by studying the averaging kernel functions on a high resolution vertical grid. The Full Width at Half Maximum (FWHM) of the averaging kernel functions are presented in Figure 2.

The FWHM values for the 1- line are around $2.5 \mathrm{~km}$ for the lower altitudes, and increase to about $6 \mathrm{~km}$ at the upper altitudes. The $9+$ line shows slightly higher FWHM values, ranging between 2.5 and $7 \mathrm{~km}$. All other lines exceed FWHM values of $10 \mathrm{~km}$ in the upper atmosphere. The small scale oscillations on the FWHM values are caused by interpolation when calculating the width of the averaging kernel function at half maximum.

In order to optimize the vertical grid, one should also examine the smoothing error of the grid, as given by Eq. 6 . The impact of a $2 \mathrm{~km}, 3 \mathrm{~km}, 4 \mathrm{~km}, 5 \mathrm{~km}$, and $6 \mathrm{~km}$ vertical retrieval grid has been investigated. No vertical correlations in the a priori covariance matrix have been introduced. A grid with no correlations assumes correlations to be smaller than the grid size, hence an increase in vertical resolution without introducing correlations between the different retrieval levels demands more information from the retrieval. The smoothing error is independent of the retrieval grid for altitudes where enough information is provided by the measurement. Results are presented in Figure 3. For resolutions where $32 \mathrm{~km}$ is not a retrieval grid point, the closest to $32 \mathrm{~km}$ is chosen as the pointing bias retrieval grid point. For resolutions where $100 \mathrm{~km}$ is not a retrieval grid point, all altitudes $<100 \mathrm{~km}$ are calculated.

A $3 \mathrm{~km}$ retrieval grid is feasible for the 1- line for altitudes ranging from 20 to $40 \mathrm{~km}$, since the introduced smoothing error is very small. A retrieval resolution of $4 \mathrm{~km}$ is possible above $40 \mathrm{~km}$ without increasing the smoothing error to $>4 \mathrm{~K}$. The $9+$ line allows a $4 \mathrm{~km}$ retrieval resolution throughout the stratosphere, and a 5 to $6 \mathrm{~km}$ resolution for the mesosphere with a smoothing error $<5 \mathrm{~K}$. All other lines allow a $4 \mathrm{~km}$ retrieval resolution for lower stratospheric altitudes. The $17+$ and $19+$ lines allow sampling of the mesosphere with a 5 to $6 \mathrm{~km}$ resolution and a smoothing error $<5 \mathrm{~K}$. Large contributions from the smoothing error for the $21+$ and $25+$ lines indicate that they are not suited for mesospheric temperature retrieval with this resolution. The measurement error for the different grids (not shown) is generally $<1 \mathrm{~K}$ for stratospheric altitudes, and about $2 \mathrm{~K}$ for mesospheric altitudes.

In the previous discussion we have not included correlations in the a priori covariance matrix between the different retrieval levels. Correlations can be calculated from radiosonde measurements or taken from NWP model output. 


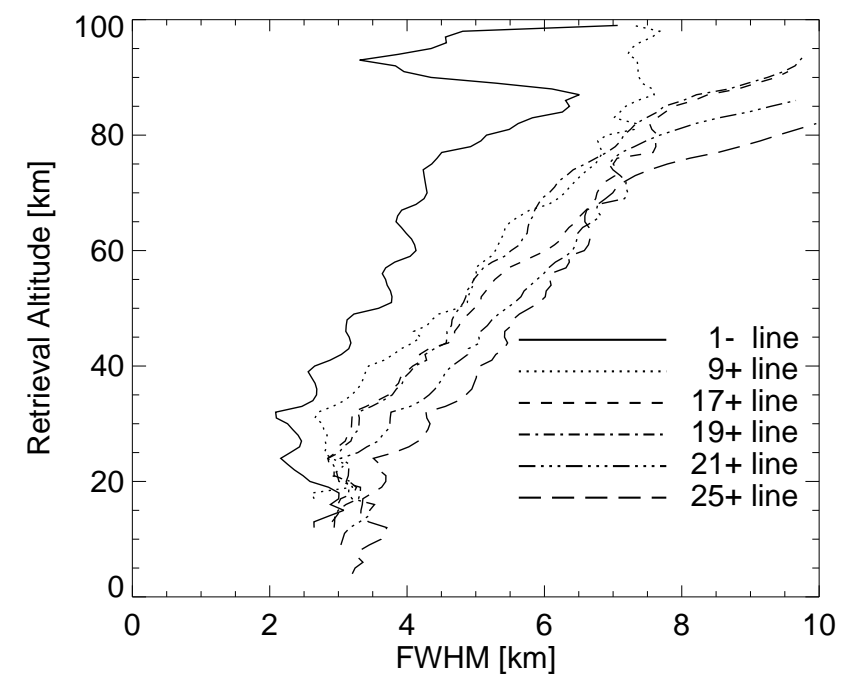

Figure 2. FWHM values of the averaging kernel functions for different oxygen lines.

This approach generally limits the accessible altitude range to $\approx 30 \mathrm{~km}$ (radiosondes) or $\approx 60 \mathrm{~km}$ (NWP models).

The impact of correlations on the total retrieval error is shown in Figure 4. Plotted are different correlation lengths, which are defi ned as the distance at which the correlations drop to $1 / e$. The equidistant $4 \mathrm{~km}$ vertical retrieval grid scenario for the $9+$ line is shown.

The $0 \mathrm{~km}$ calculation corresponds to no correlations. Increasing the correlation length decreases the estimated error in the retrieval, especially in regions with higher total retrieval errors. Due to sparse measurement coverage in the mesosphere however it is diffi cult to determine the appropriate correlation length. We therefore adopt an approach with no correlations between the different vertical retrieval levels.

We consequently use a $4 \mathrm{~km}$ equidistant vertical grid covering the altitude interval from 0 to $100 \mathrm{~km}$ with no correlations in the a priori covariance matrix for a comparison of the two main lines. This nominal scenario ensures the highest possible resolution in the mesosphere and a moderate one in the stratosphere.

The averaging kernel functions for the nominal scenario of all lines are plotted in Figure 5. Additionally, the sum of the averaging kernels is included. The $1-$ line provides the best averaging kernels throughout the mesosphere. But good mesospheric coverage is provided by the $9+, 17+$, and $19+$ line as well. Weaker lines provide more informations at lower altitudes, as can be seen for the $21+$, and $25+$ line.

\subsection{Weighting Function Elements}

Information about the temperature at a specifi $\mathrm{c}$ retrieval altitude comes from different instrument fi lters which can be visualized with the aid of the weighting function matrix K. Figure 6 shows the normalized elements of the $\mathbf{K}$ matrix for the nominal scenario of the $9+$ line. Contours show the temperature weighting function elements for a specifi $\mathrm{c}$ fi lter, where the filter center frequency is given in the left hand corner of the plot (fi rst 5 plots). Additionally, the normalized elements of $\mathbf{K}$ for the pointing bias are shown (last plot).

The upper left hand plot shows one of the outermost fi lter with a center frequency at $0.4815 \mathrm{GHz}$. This fi lter primarily provides information about retrieval altitudes around $28 \mathrm{~km}$. The information is embedded in measurement tangent altitudes ranging from 0 to $40 \mathrm{~km}$. Measurement tangent altitudes below $28 \mathrm{~km}$ provide information about the temperature around $28 \mathrm{~km}$ because this filter gets opaque around $25 \mathrm{~km}$. Measurement tangent altitudes above $28 \mathrm{~km}$ provide information about the temperature around $28 \mathrm{~km}$ due to the antenna sidelobes. The upper middle plot shows a fi lter closer to the center, providing temperature information around $36 \mathrm{~km}$ and stretching in measurement tangent altitudes from 0 to $60 \mathrm{~km}$. Almost all measurement tangent altitudes provide information about the temperature around $80 \mathrm{~km}$ for a fi lter at the center of the line (lower middle plot), since this filter gets opaque for measurement tangent altitudes around $80 \mathrm{~km}$ (see Figure 1). Above $85 \mathrm{~km}$, where 

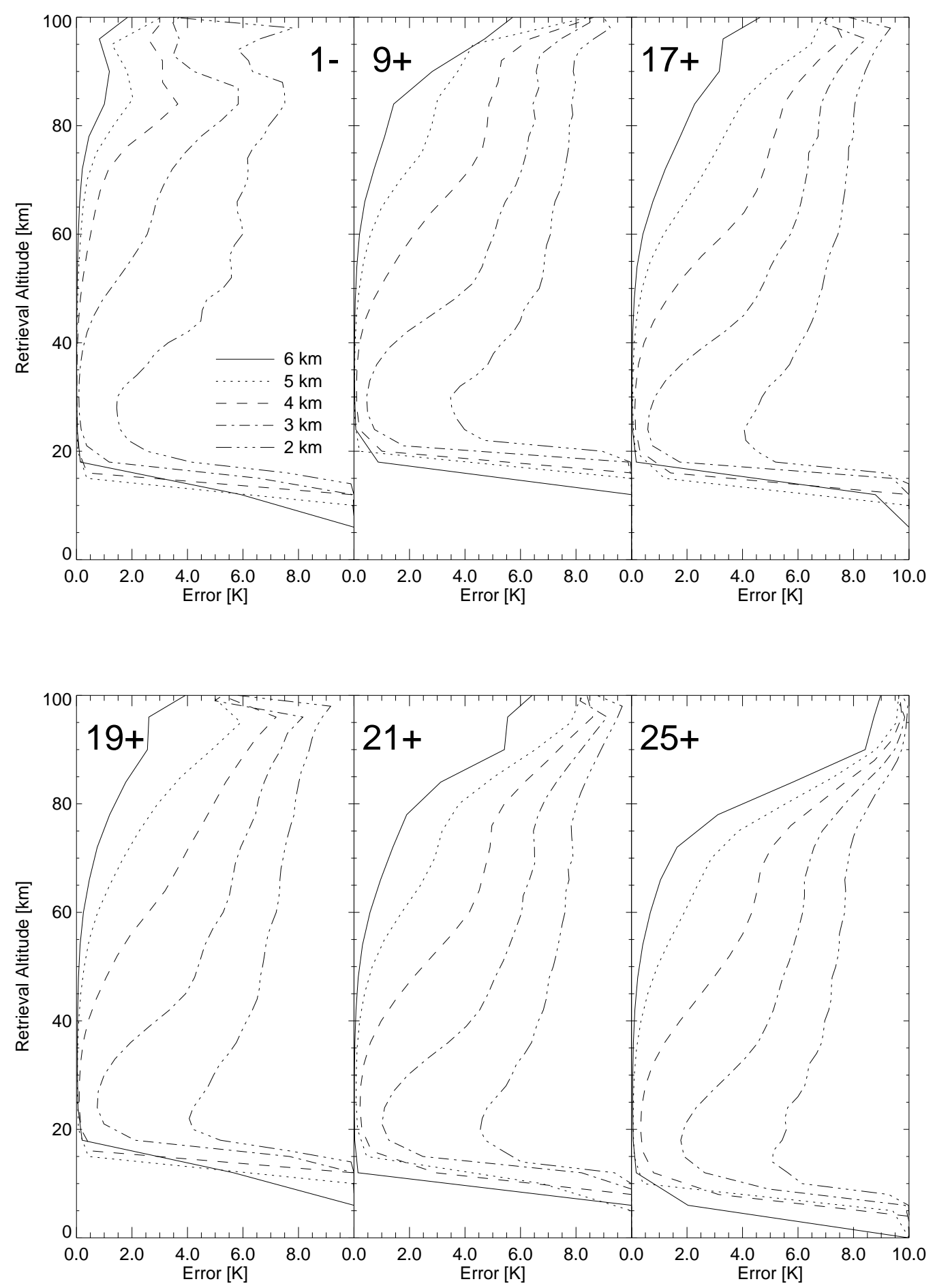

Figure 3. Smoothing error profi les for different vertical retrieval grids. 


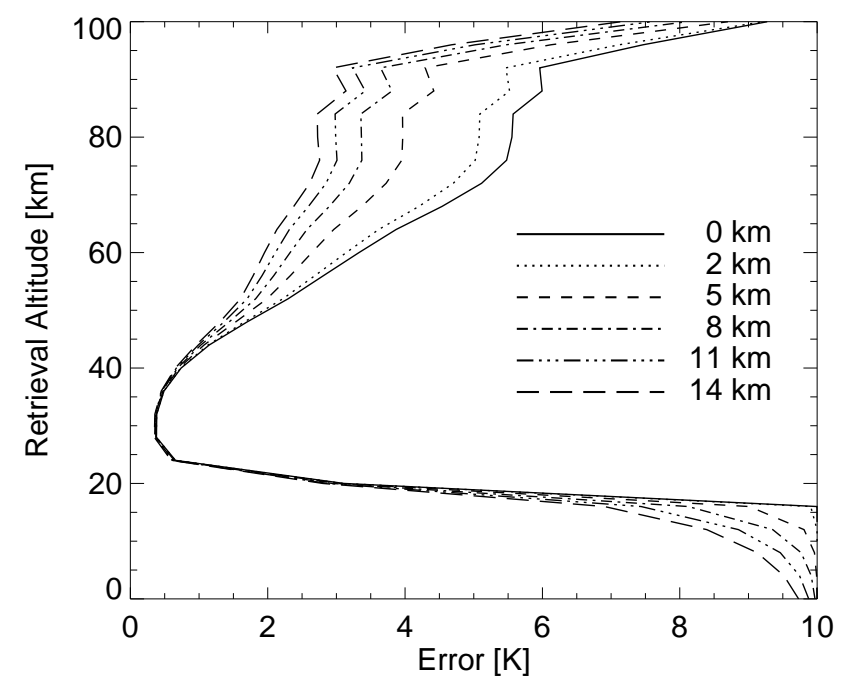

Figure 4. Total retrieval error profi les for the $61.15 \mathrm{GHz}$ line for different correlation lengths in the a priori covariance matrix.

this fi lter is not opaque, information about the temperature at lower retrieval altitudes is provided via the antenna sidelobes.

The lower right hand side plot in Figure 6 shows the normalized weighting function matrix elements of the pointing bias retrieval. Information about the pointing bias is provided by measurement tangent altitudes around the pointing bias grid point for the outermost frequencies. The measurement tangent altitudes providing information move higher up for frequencies closer to the center frequency, indicating that the pointing bias retrieval information comes mainly from non-opaque fi lters.

These basic features of the weighting function matrix are identical for all lines.

\subsection{General Retrieval Capabilities}

Depending on strength and position, different oxygen lines are suited best for different altitude regions. The different investigated oxygen lines are listed in Table 1. The total retrieval error profi les are given in Figure 7 for the nominal scenario. The features discussed above in connection with the averaging kernel functions can be seen here as well. Weak lines such as the $25+$ transition allow temperature profi le determination down to tangent altitudes of about $10 \mathrm{~km}$, while strong lines such as the $9+$ one have a lower limit of about $20 \mathrm{~km}$. The presence of additional transitions nearby also affects the range over which temperature can be deter- mined. The isolated position of the $1-$ line allows temperature profi le determination from tangent altitudes of $16 \mathrm{~km}$ upward, despite the fact that the intensities of the $9+$ line and the 1- are comparable.

The total retrieval error of the pointing bias retrieval is presented in Table 2. The pointing bias retrieval is possible with very high accuracy, where the accuracy reflects the detectable altitude region of the line (compare to Figure 5).

\subsection{Instrumental Parameters}

The temperature retrieval capabilities are affected by several instrumental parameters, where the following are investigated for the two main lines:

- System noise temperature

- Antenna pattern Full Width at Half Maximum

- Total coverage of line

- Filter widths

The impact on the pointing bias retrieval is insignifi cant and thus not included in the discussion.

4.4.1. System Noise Temperature The nominal scenario assumes a SSB receiver with a system noise temperature of $1000 \mathrm{~K}$. A cooled receiver such as the ODIN-SMR can achieve a system noise temperature of $600 \mathrm{~K}$ for SSB measurements (D. Murtagh, Mission Scientist at Dept. of 

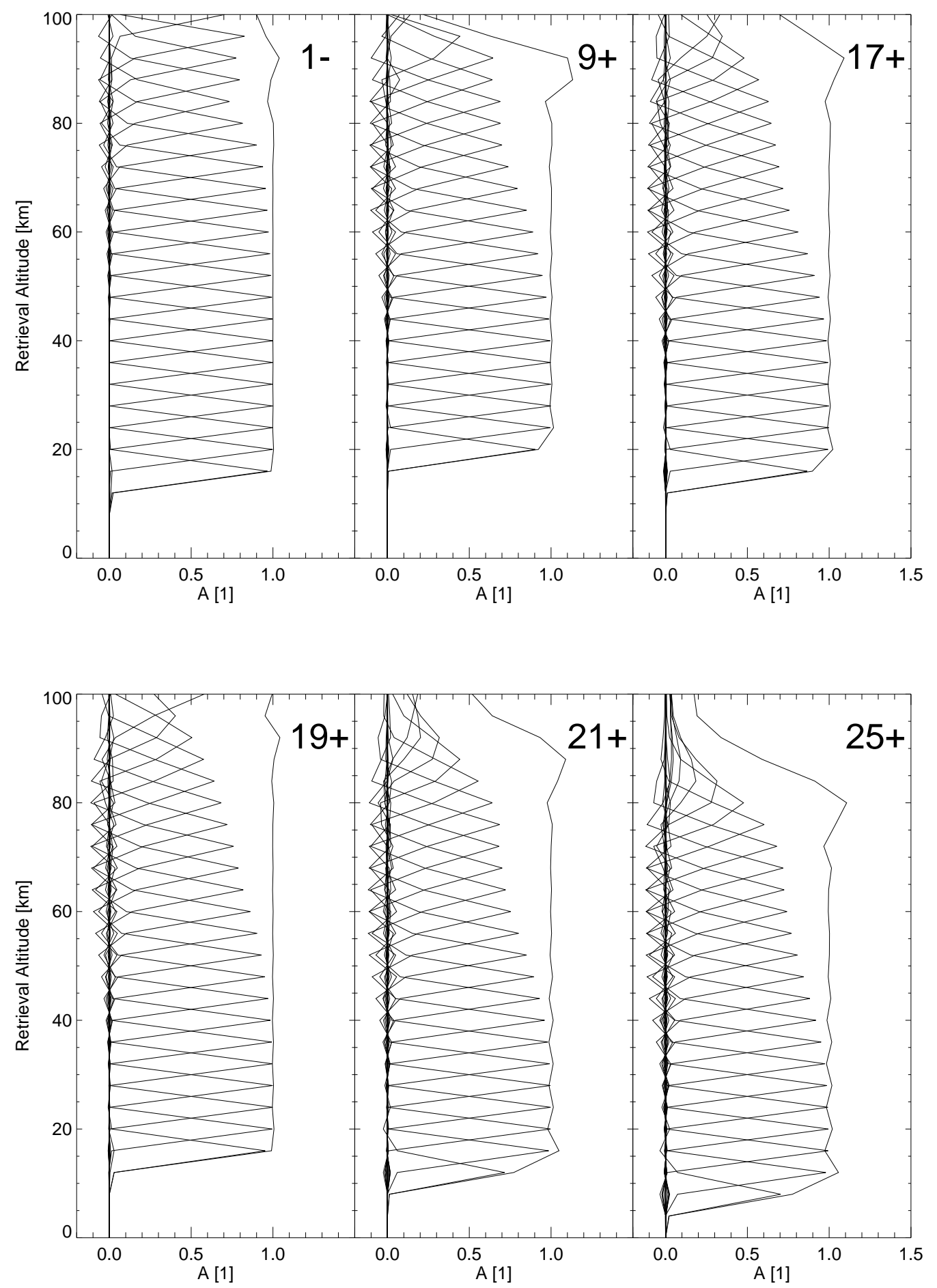

Figure 5. Averaging kernel functions and sum for different oxygen lines. 

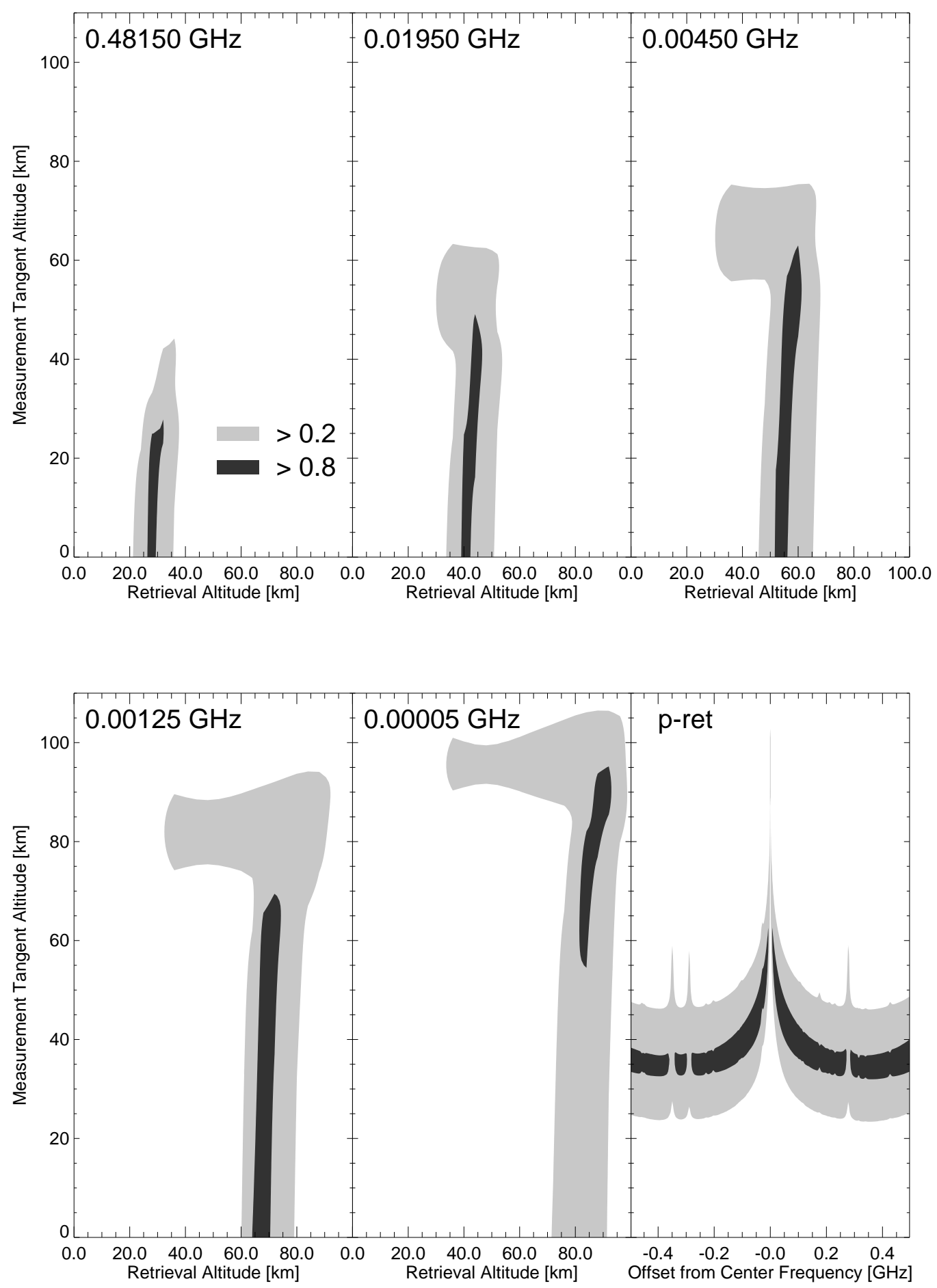

Figure 6. First 5 plots: Normalized temperature weighting function matrix elements for the $61.15 \mathrm{GHz}$ line for 5 particular fi lters situated at the given center frequency. Last plot: Normalized pointing bias weighting function elements. 


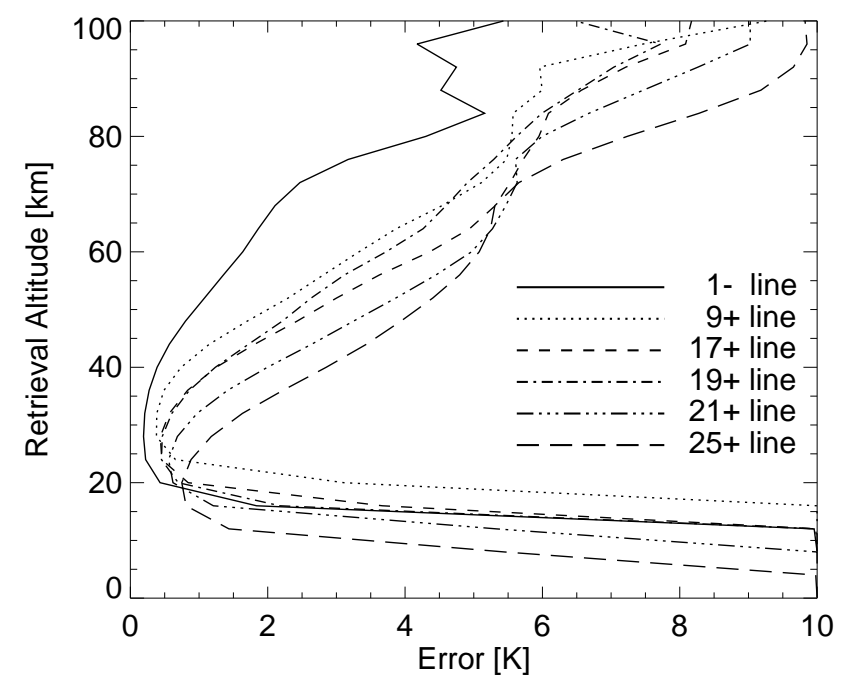

Figure 7. Total retrieval error profi les for different oxygen lines.

Table 2. Total retrieval errors on pointing bias expressed in relative pressure units and corresponding geometric tangent altitude (TA) errors.

\begin{tabular}{lcc}
\hline Line & Pressure Error [\%] & TA Error $[\mathrm{km}]$ \\
\hline $9+$ & 0.025 & 0.002 \\
$1-$ & 0.019 & 0.001 \\
$17+$ & 0.030 & 0.002 \\
$19+$ & 0.031 & 0.002 \\
$21+$ & 0.039 & 0.003 \\
$25+$ & 0.053 & 0.004 \\
\hline
\end{tabular}


Radio and Space Science, Chalmers University of Technology, personal communication, 2001), while the EOS-MLS system noise temperature is given as $1500 \mathrm{~K}$ [Waters, 1999].

The total retrieval error profi les for different system noise temperatures are shown in Figure 8. Reductions in system noise temperature are particularly important for retrievals at upper stratospheric and mesospheric altitudes. As can be seen from Eq. 9, there is a direct relationship between the integration time and the system noise temperature. A reduction of the noise temperature by a factor of 0.5 leads to the same benefi $t$ as an increase of the integration time by a factor of 4 .

4.4.2. Antenna Pattern FWHM The Full Width at Half Maximum (FWHM) of the beam width for most microwave antennas can be calculated with an accuracy of about $10 \%$ according to Eq. 1.33 in Janssen [1993]:

$$
\theta_{\mathrm{FWHM}}=1.5 \lambda / D_{a}
$$

where $\theta_{\mathrm{FWHM}}$ gives the beam width of the antenna, $D_{a}$ the diameter, and $\lambda$ the corresponding observation wavelength.

The MASTER antenna pattern is given at $200 \mathrm{GHz}$ with a FWHM of about $3.4 \mathrm{~km}$ [Reburn et al., 1999], slightly better than described by Eq. 10 which would result in a FWHM of about $3.7 \mathrm{~km}$ for an antenna with a diameter of $2 \mathrm{~m}$. Eq. 10 was used to scale the antenna to the observation frequency at $61.15 \mathrm{GHz}$ and $118.75 \mathrm{GHz}$, where FWHM of $11.1 \mathrm{~km}$ and $5.8 \mathrm{~km}$, respectively, are found. The ODIN-SMR has an antenna diameter of $1.1 \mathrm{~m}$ [Räisänen et al., 1996] in an orbit of about $600 \mathrm{~km}$, which according to Eq. 10 results in a FWHM value of about $9 \mathrm{~km}$ for the $1-$ line at the tangent point. The EOS-MLS instrument has a $1.6 \mathrm{~m}$ antenna and a FWHM of $6.5 \mathrm{~km}$ is given in Waters [1999] at the tangent point for the 1- line.

The effect of different antenna beam widths is shown in Figure 9, where the nominal FWHM of the antenna pattern has been scaled to the values given in the legend. Minor improvements of $10 \%$ in the FWHM value of the beam width produce only small improvements in the total retrieval error profi le, substantial reductions are required to improve the error of the retrieved temperature profi le by $1 \mathrm{~K}$ for the $9+$ line. The major improvement is found at mesospheric altitudes, because strong tropospheric emissions are reduced with decreasing beam width. The $1-$ line is less sensitive to the antenna beam width, owing to the lower influence of tropospheric emissions due to the smaller beam width.

Even though the intensity of the the 1- line is lower than that one of the 9+ line [Liebe and Hufford, 1989], it still has a lower total retrieval error for all altitudes due to the frequency dependence of Eq. 10, as can be seen in Figure 9.

4.4.3. Total Coverage of Line The nominal scenario assumes a band of $1 \mathrm{GHz}$ centered on the line of interest.
The lowest possible altitude down to which temperature information can be derived is a function of the total bandwidth, since pressure broadening of the line leads to an increasing line width at lower altitudes and the transmission becomes opaque near line center.

The ODIN-SMR instrument has a total bandwidth of $1 \mathrm{GHz}$ (D. Murtagh, Mission Scientist at Dept. of Radio and Space Science, Chalmers University of Technology, personal communication, 2001), while the EOS-MLS instrument has a $1.3 \mathrm{GHz}$ coverage around the line center. Additionally, EOS-MLS observes the 1- line wings with very wide fi lters $(0.5 \mathrm{GHz})$ to obtain information at altitudes below about $15 \mathrm{~km}$ [Waters, 1999].

The results for different band widths are presented in Figure 10. Reduction of the band width has only a small effect on the $9+$ line, the $0.8 \mathrm{GHz}$ band is almost identical to the $1 \mathrm{GHz}$ band. Only the lowermost detectable altitudes are affected by a reduction to $0.4 \mathrm{GHz}$. The $1-$ line, on the other hand, is sensitive to the band width. A further increase in band width will improve the retrieval capabilities at lower altitudes.

4.4.4. Filter Widths The nominal scenario assumes coverage of the line wings with $3 \mathrm{MHz}$ fi lter, and around the line center with $0.1 \mathrm{MHz}$ fi lter. The retrieval capability is determined by the wide fi lters at lower altitudes and by the narrow fi lters at mesospheric altitudes.

The ODIN-SMR has a coverage of $1.1 \mathrm{MHz}$ fi lters and optional a high resolution measurement with fi lters of $0.15 \mathrm{MHz}$ width [Eriksson, 1999]. EOS-MLS provides fi lter widths between $6 \mathrm{MHz}$ and about $100 \mathrm{MHz}$ to cover the line wings, and additionally fi lters within the center region with a width of $0.2 \mathrm{MHz}$ [Waters, 1999].

Figure 11 shows the impact of different fi lter widths. All fi lters are assumed to have a response curve of 1 within the covered frequency interval, and 0 outside. The wide fi lter calculations are without coverage of $\pm 20 \mathrm{MHz}$ around the center region to avoid different overlap of the filters with the center region, which would make the interpretation of the plot more diffi cult. Only the center fi lter channels were modifi ed for all fi ne fi lter calculations, otherwise the nominal scenario is employed.

The wide fi lters have only a minor impact for both the 1and $9+$ line. Only in the case of the $40 \mathrm{MHz}$ fi lters is there some effect on the total retrieval error profi le of the $9+$ line.

The fi ne fi lters, on the other hand, affect mainly the temperature retrieval at altitudes above about $60 \mathrm{~km}$ (see Figure 6). The $9+$ line is less affected by the actual width of the fi ne filter, a reduction of the resolution to $800 \mathrm{kHz}$ is still acceptable. The impact of the resolution is much more severe on the $1-$ line, since the $1-$ line splits up into only 3 

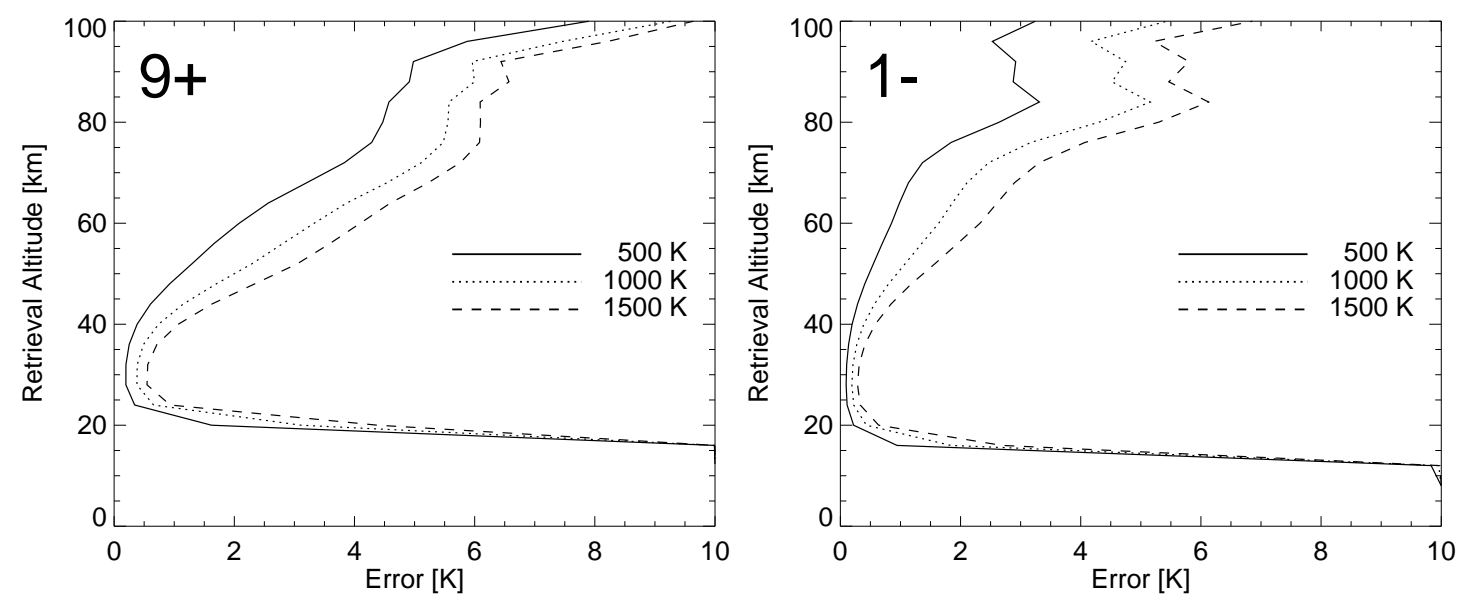

Figure 8. Total retrieval error profi les for different system noise temperatures for the $61.15 \mathrm{GHz}$ line (left) and the $118.75 \mathrm{GHz}$ line (right).
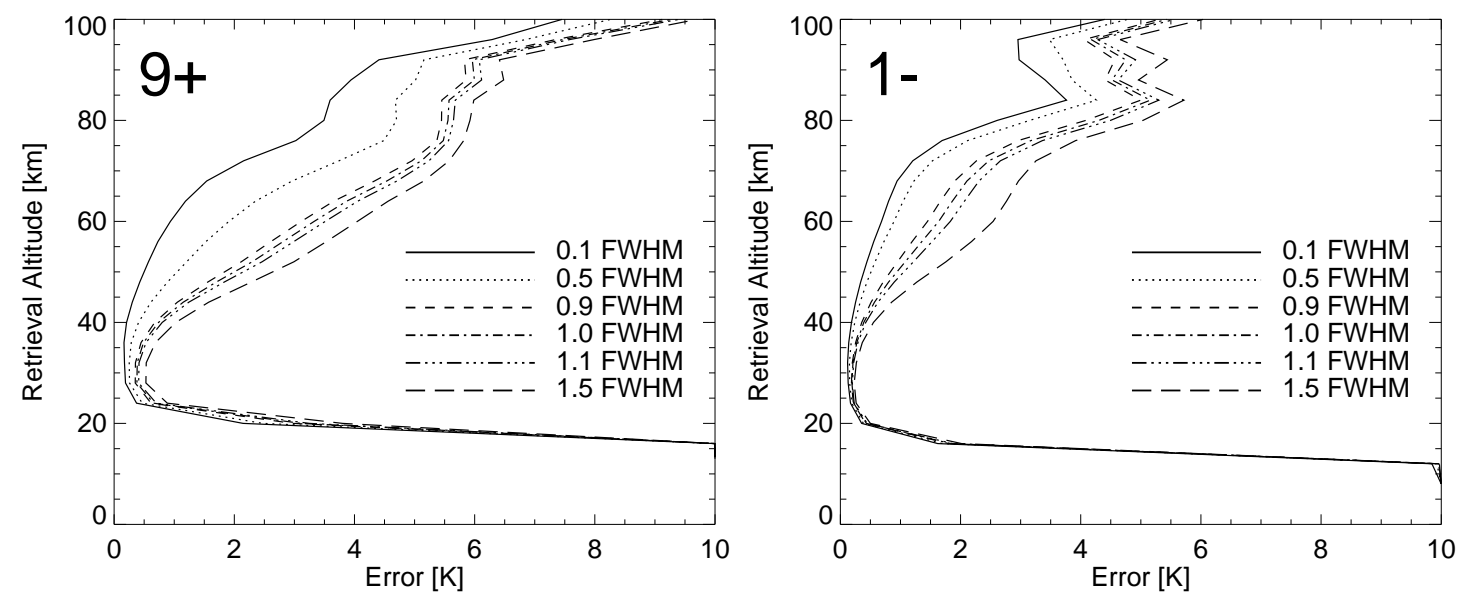

Figure 9. Total retrieval error profi les for different antenna beam widths for the $61.15 \mathrm{GHz}$ line (left) and the $118.75 \mathrm{GHz}$ line (right). 

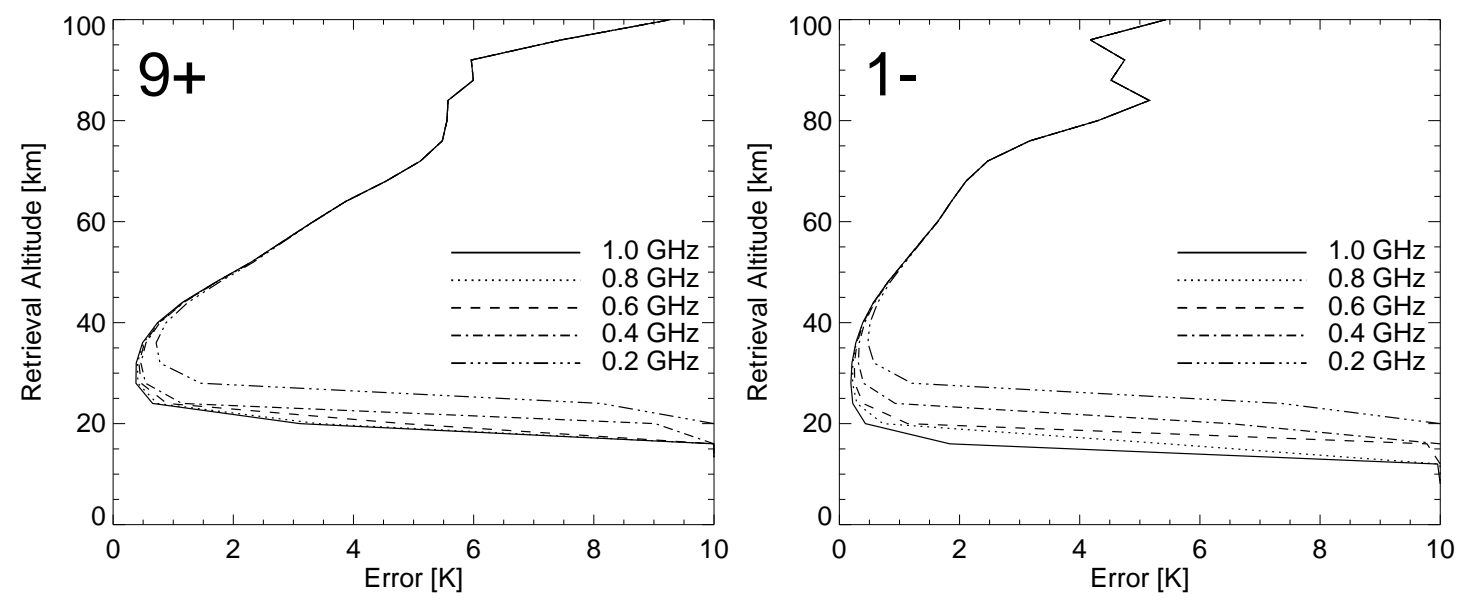

Figure 10. Total retrieval error profi les for different band widths for the $61.15 \mathrm{GHz}$ line (left) and the $118.75 \mathrm{GHz}$ line (right).

transmissions in the Earth's magnetic fi eld, while the the $9+$ line splits up into 57. Thus narrow fi lters can sample the individual transmissions of the $1-$ line, which is not possible for the $9+$ line because the 57 lines overlap to form a cluster (see Figure 1).

\subsection{Spectroscopic Errors}

The forward model used here can read spectroscopic line parameters from several spectroscopic databases, such as the JPL catalog [Pickett and Poynter, 1985], or HITRAN [Rothman et al., 1992]. The standard spectroscopic parameters do not suffi ciently describe the oxygen spectrum near $60 \mathrm{GHz}$, as for example discussed by Rosenkranz [1988]. Thus updated line parameters as given by Liebe et al. [1992] are included.

In total, fi ve spectroscopic parameters are used to describe the line: the line center frequency, the line intensity, the line width, and two overlap parameters, where we chose the $y$ and $v$ parameter, as given by Rosenkranz [1993].

The 9+ line is situated in between two other strong oxygen lines, the $7+$ line at $60.43 \mathrm{GHz}$, and the $11+$ line at $61.80 \mathrm{GHz}$. Thus it is necessary to include the spectroscopic parameters of these lines in the investigation. The 1- line is isolated at $118.75 \mathrm{GHz}$, and only the spectroscopic parameters of the line itself were investigated.

Figure 12 shows the resulting model parameter error in the retrieved temperature for the $9+$ line caused by a $5 \%$ error in the corresponding spectroscopic parameter. The error is calculated from Eq. 8, which assumes a linear relationship between an error in the spectroscopic parameters and the resulting model error in the retrieval parameters.

The main impact on the temperature retrieval is caused by errors in the spectroscopic parameters of the observed line. Only errors in intensity and line width have a signifi cant impact, errors in the center frequency and the overlap parameters are minor (not shown). Overlap is proportional to pressure [Rosenkranz, 1993], and its main influence is found at altitudes below about $16 \mathrm{~km}$, where the $9+$ line is insensitive to temperature observations.

A pointing bias is also retrieved, but improvements in the AOCS beyond the current specifi cations could make the pointing bias retrieval redundant. However, the impact of spectroscopic errors on the temperature retrieval are greatly increased in the absence of a simultaneous pointing bias retrieval (see Figure 12 right hand side plots). Thus, when a pointing bias is retrieved an error in a spectroscopic parameter is partially compensated for by an offset in the pointing. Nevertheless, temperature model parameter errors of several Kelvin can occur even when the pointing bias is retrieved.

The impact of spectroscopic errors for the $1-$ line is shown in Figure 13. The nominal retrieval scenario with pointing bias retrieval shows much lower model parameter errors caused by uncertainties in the spectroscopic parameters in comparison to the $9+$ line. As is the case for the $9+$ line the main effect of an error in the spectroscopic parameters is to alter the retrieved pointing bias. This can be seen in Figure 13 (right hand side), spectroscopic parameter errors will cause a large temperature error if the pointing bias retrieval is omitted. Errors in the center frequency and the 

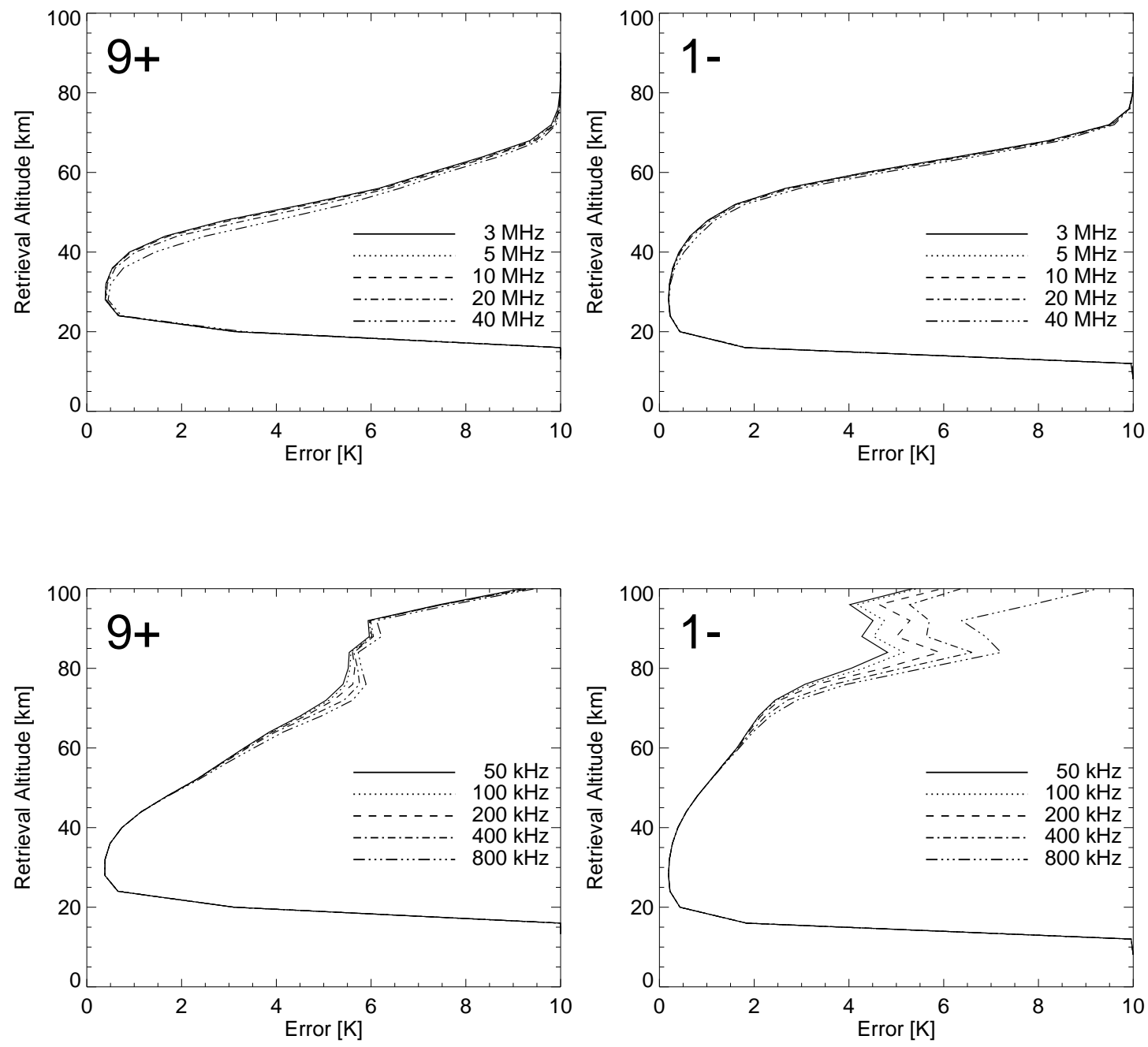

Figure 11. Total retrieval error profi les for different fi lter widths for the $61.15 \mathrm{GHz}$ line (left panels) and the $118.75 \mathrm{GHz}$ line (right panels). The upper plots show the error profi les for the wide fi lter coverage, the lower ones for the fi ne fi lter coverage around the line center. 


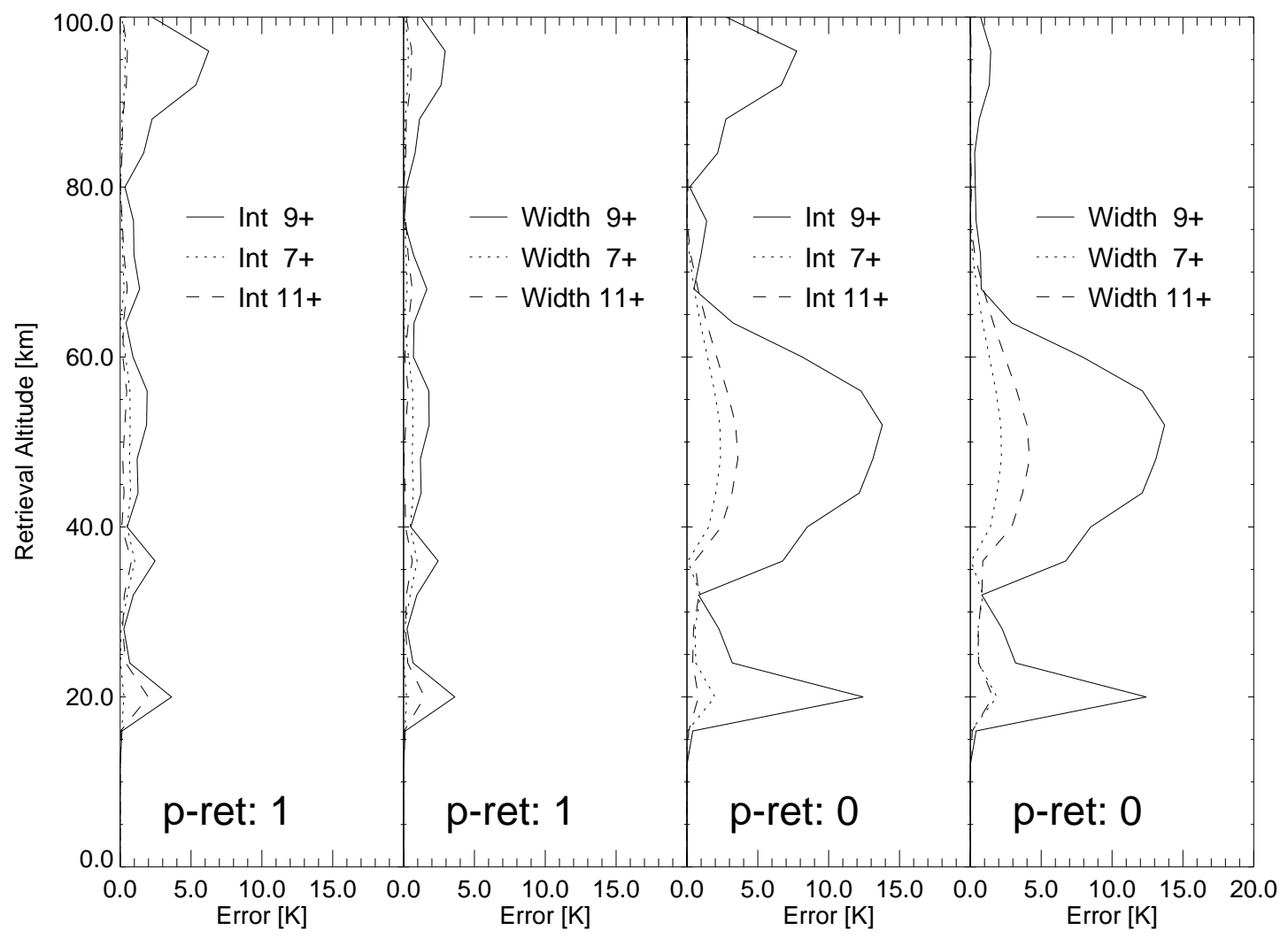

Figure 12. Model parameter error profi les of temperature for the $61.15 \mathrm{GHz}$ line caused by an error in the spectroscopic parameter. The left two panels show the result for the nominal scenario, where the temperature profi le and a pointing bias is retrieved (p-ret: 1). The right hand side shows the results for a temperature retrieval only situation (p-ret: 0 ). 


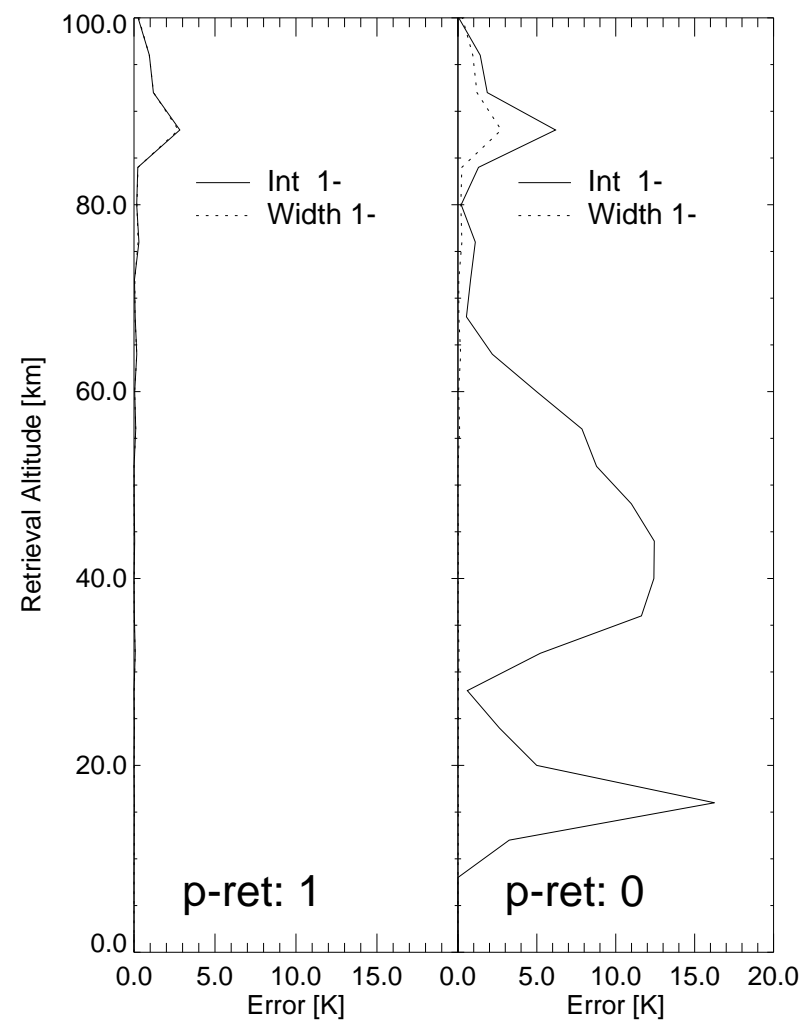

Figure 13. Model parameter error profi les of temperature for the $118.75 \mathrm{GHz}$ line caused by an error in the spectroscopic parameter. The left panel shows the result for the nominal scenario, where the temperature profi le and a pointing bias is retrieved (p-ret: 1). The right hand side shows the results for a temperature retrieval only (p-ret: 0 ). 
overlap parameters have a minor impact (not shown).

The temperature retrieval from the $9+$ line shows a higher sensitivity to errors in the spectroscopic parameters for the nominal scenario than that from the $1-$ line. The retrieved pointing bias value is to some extent determined by the neighboring oxygen lines since these provide pressure information and pointing bias information comes from the pressure broadening of the lines. Thus errors show up directly in the model parameter error of the temperature retrieval. The 1- line is isolated, and errors in spectroscopic parameters will mainly lead to a model parameter error in the retrieved pointing bias, while the temperature is less affected. This shows up as well in iterative retrievals when using wrong spectroscopic parameters, where the retrieval adjusts the pointing bias for the 1- line more strongly.

The actual shape of the model parameter error profi le reflects different information sources. At the lower altitudes information comes primarily from opaque channels. The number of opaque channels decreases with increasing altitude, and at about $30 \mathrm{~km}$ the information begins to be embedded in the lineshape, reaching a maximum at about $50 \mathrm{~km}$. The model parameter error decreases between about $50 \mathrm{~km}$ and $80 \mathrm{~km}$ as a result of the decreasing line width with altitude. Above $80 \mathrm{~km}$ opaque channels in the center provide information and an increasing model parameter error is found.

Note that the model parameter error patterns for intensity and width look very similar for the $1-$ and $9+$ line up to altitudes of about $80 \mathrm{~km}$. Above this altitude, an erroneous intensity affects all observed brightness temperatures, resulting in large model parameter errors in the retrieved temperatures. An erroneous width on the other hand leads only to a different distribution of the intensity over a frequency interval, because the line shape is normalized. Since the fi lters integrate over a large fraction of this frequency interval, errors in the line width are less severe. This behavior is not seen at lower altitudes, since the filters are covering only a fraction of the observed line here.

The impact of an uncertainty in the different spectroscopic parameters on the retrieved pointing bias is shown in Table 3 for the $9+$ line, and in Table 4 for the $1-$ line, along with the corresponding relative error in pressure units. A model parameter error of $1 \%$ in relative pressure units corresponds to an error in tangent altitude of almost $0.1 \mathrm{~km}$. As mentioned above, the impact on pointing bias is larger for the 1- line. Largest model parameter errors are caused by uncertainties in intensity and width of the line. An uncertainty in the line center frequency and the overlap parameters is not signifi cant.

Current NWP models achieve a resolution of up to $1 \mathrm{~km}$ in the upper troposphere [ECMWF, 2000], thus pointing bias errors introduced by spectroscopic uncertainties could affect the assimilation of data obtained from these kind of observations.

\section{Conclusions}

We investigate several instrumental parameters to observe molecular oxygen emission lines in the microwave for the determination of the atmospheric temperature profi le and an instrumental pointing bias. Additionally, the impact of spectroscopic errors is addressed. The main focus of this investigation is on the $9+$ line at $61.15 \mathrm{GHz}$ within the cluster of oxygen lines around $60 \mathrm{GHz}$, and the isolated $1-$ line at $118.75 \mathrm{GHz}$. We use a polarized radiative transfer model that includes the effect of Zeeman splitting due to the Earth's magnetic fi eld to simulate measurements taken from these oxygen lines.

The underlying instrumental scenario was based on the MASTER instrument, a microwave limb sounder currently investigated by the European Space Agency. The atmospheric scenario chosen was a mid-latitude summer one. The temperature profi le was retrieved between 0 and $100 \mathrm{~km}$.

A thorough investigation into the possible vertical retrieval grid resolution was performed. A $3 \mathrm{~km}(4 \mathrm{~km})$ resolution for lower altitudes, and a $4 \mathrm{~km}(5 \mathrm{~km})$ one for upper altitudes is possible with the $1-(9+)$ line. For a comparison of the two main lines, a $4 \mathrm{~km}$ grid without correlations in the a priori covariance matrix is best suited.

Even though the $9+$ line within the $60 \mathrm{GHz}$ cluster has a higher intensity, temperature retrieval from the $1-$ line at $118.75 \mathrm{GHz}$ is superior at all altitudes. At low altitudes this is caused by the isolated position of the $1-$ line. The $9+$ line is surrounded by two other strong oxygen line and the signal becomes opaque at low altitudes. At high altitudes the higher observation frequency leads to better results, since the antenna beam width is inversely proportional to the wavelength of the observation.

Oxygen lines within the cluster with slightly lower intensities are comparable to the $9+$ line at mesospheric altitudes, and allow temperature retrievals at lower altitudes. The use of oxygen lines with very low intensities moves the accessible altitudes further down, but is disadvantageous for mesospheric altitudes. Pointing bias retrieval with a very high accuracy is possible from all investigated lines.

Improvements in the system noise temperature are most advantageous at mesospheric altitudes, a system noise temperature of $500 \mathrm{~K}$ allows temperature retrieval from the 1line with a total retrieval error below $3 \mathrm{~K}$ for stratospheric and mesospheric altitudes.

A $10 \%$ variation in the antenna beam width has only a minor effect on the retrieval capability. The use of the 1line for temperature sounding is advantageous since for a 
Table 3. Model parameter errors on pointing bias caused by uncertainties in the spectroscopic parameters (9+ line), expressed in relative pressure units and corresponding geometric tangent altitude (TA) errors.

\begin{tabular}{lcr}
\hline \multicolumn{1}{c}{ Parameter } & Pressure Error [\%] & TA Error [km] \\
\hline Intensity 9+ & 1.25444 & 0.089 \\
Intensity 7+ & 0.31860 & 0.023 \\
Intensity 11+ & 0.39711 & 0.028 \\
Width 9+ & 1.25269 & 0.089 \\
Width 7+ & 0.29548 & 0.021 \\
Width 11+ & 0.43671 & 0.031 \\
Overlap y 9+ & 0.00022 & $1.5 \mathrm{E}-5$ \\
Overlap y 7+ & 0.02717 & 0.002 \\
Overlap y 11+ & 0.01403 & 0.001 \\
Overlap v 9+ & 0.00009 & $6.2 \mathrm{E}-6$ \\
Overlap v 7+ & 0.00457 & $3.3 \mathrm{E}-4$ \\
Overlap v 11+ & 0.01092 & $7.8 \mathrm{E}-4$ \\
Center Freq. 9+ & 0.00021 & $1.5 \mathrm{E}-5$ \\
Center Freq. 7+ & 0.00035 & $2.5 \mathrm{E}-5$ \\
Center Freq. 11+ & 0.00059 & $4.2 \mathrm{E}-5$ \\
\hline
\end{tabular}

Table 4. Model parameter errors on pointing bias caused by uncertainties in the spectroscopic parameters (1- line), expressed in relative pressure units and corresponding geometric tangent altitude (TA) errors.

\begin{tabular}{lcr}
\hline \multicolumn{1}{c}{ Parameter } & Pressure Error [\%] & TA Error [km] \\
\hline Intensity 1- & 2.46891 & 0.176 \\
Width 1- & 2.44919 & 0.174 \\
Overlap y 1- & 0.00015 & $1.0 \mathrm{E}-5$ \\
Overlap v 1- & 0.00001 & $6.9 \mathrm{E}-7$ \\
Center Freq. 1- & 0.00004 & $3.4 \mathrm{E}-6$ \\
\hline
\end{tabular}


given antenna size the higher observation frequency reduces the beam width by $\approx 50 \%$ relative to the $9+$ line, and thus improves retrieval sensitivity.

The nominal scenario assumes a total bandwidth of $1 \mathrm{GHz}$, centered on the line of interest. A decrease of the total covered bandwidth from $1 \mathrm{GHz}$ to $0.8 \mathrm{GHz}$ is possible for the $9+$ line without loss of information. Any decrease in the bandwidth of the 1- line affects the retrieval accuracy because due to its isolated position, an increase of the bandwidth allows access of lower altitudes.

The impact of different fi lter widths was assessed. For the wide fi lters, used for the coverage of the line wings, a reduction in resolution from $3 \mathrm{MHz}$ to $40 \mathrm{MHz}$ for the 1line and to $20 \mathrm{MHz}$ for the $9+$ line causes only a minor decrease in sensitivity. The fi ne fi lters, used for coverage of the line center, allow a reduction from $100 \mathrm{kHz}$ to $800 \mathrm{kHz}$ for the $9+$ line, with only minor decreases in sensitivity. The 1- line is affected by the choice of fi ne fi lters at high altitudes; a $100 \mathrm{kHz}$ resolution proves to be suffi cient for mesospheric temperature retrievals, an increase of the resolution to $50 \mathrm{kHz}$ improves the sensitivity only slightly.

Errors in the spectroscopic parameters of $5 \%$ can cause errors of up to several Kelvin in the retrieved temperatures. The error is caused mainly by errors in the intensity and the width parameter of the primary line being measured, neighboring lines have only a minor influence. For the $9+$ line errors in the spectroscopic parameters result in model parameter errors of up to $6 \mathrm{~K}$ at all altitudes where the observation is sensitive to temperature. The 1- line is only affected at altitudes above $80 \mathrm{~km}$.

The 1- and 9+ line show a different behavior when pointing bias is retrieved. The error in the temperature retrieval due to errors in the spectroscopic parameters is much larger for both lines when we do not retrieve the pointing bias. Errors of up to $15 \mathrm{~K}$ can be found. An uncertainty in the spectroscopic parameter is compensated by altering the retrieved pointing bias in calculations where pointing bias is retrieved. The 9+ line pointing bias retrieval is altered less, hence the temperature retrieval is more severely affected.

A simultaneous pointing bias retrieval is highly recommended, since it can compensate for erroneous spectroscopic parameters. However, the pointing bias error introduced by spectroscopic uncertainties could limit the assimilation into a NWP model of temperature profi les derived from this kind of observations. The pointing bias error introduced by an erroneous intensity is similar in magnitude to that introduced by width: around $170 \mathrm{~m}$ for the 1- line, and about $90 \mathrm{~m}$ for the $9+$ line. Current NWP models achieve a resolution of about $1 \mathrm{~km}$ in the upper troposphere.

Overall, the 1- line shows better retrieval capabilities.
Additionally, it is computationally easier to work with, because it splits up into fewer lines in the Earth's magnetic fi eld, thus requiring fewer calculations of the line shape. Very accurate spectroscopic parameter measurements are highly desirable to improve retrieval capabilities.

Acknowledgments. The authors wish to thank Dr. Gerald Nedoluha for thorough proof reading and a lot of helpful comments on the manuscript, Dr. Donal Murtagh for providing information about the ODIN-SMR instrument, and Dr. J"org Langen for information regarding the MASTER instrument. Furthermore, the authors wish to acknowledge funding through the German research projects SMILES (DLR 50EE9815), UTH-MOS (GSF 07ATC04), and by an Internal Government Study for the Integrated Program Offi ce of the National Polar-orbiting Operational Environmental Satellite System.

\section{References}

Barath, F., et al., The Upper Atmosphere Research Satellite Microwave Limb Sounder, J. Geophys. Res., 98, 10,751-10,762, 1993.

Barraclough, D., International Geomagnetic Reference Field: The fourth generation, Phys. Earth Planet. Inter., 48, 279-292, 1986.

Bauer, A., et al., Study on a spectroscopic data base for millimeter and submillimeter wavelengths, fi nal report, Tech. rep., ESAESTEC / Contract No 11581 / 95 / NL / CN, 1998.

Croskey, C. L., et al., The Millimeter Wave Atmospheric Sounder (MAS): A Shuttle-based remote sensing experiment, IEEE Trans. Geosci. Remote Sens., 40, 1090-1100, 1992.

ECMWF, Technical information of the ECMWF data archive, http://www.ecmwf.int/services/data/technical/other.html, 2000.

Eriksson, P., Microwave radiometric observations of the middle atmosphere: Simulations and inversions, Ph.D. thesis, Chalmers University of Technology, 1999.

Fishbein, E., et al., Validation of UARS Microwave Limb Sounder temperature and pressure measurements, J. Geophys. Res., 101, 9,983-10,016, 1996.

Gordy, W., and R. Cook, Microwave Molecular Spectra, WileyInterscience, New York, 1970.

Janssen, M., An introduction to the passive microwave remote sensing of atmospheres, in Atmospheric Remote Sensing by Microwave Radiometry, edited by M. Janssen, chap. 1, pp. 1-35, John Wiley, New York, 1993.

Liebe, H., and G. Hufford, Millimeter-wave propagation in the mesosphere, Tech. Rep. NTIA 89-249, U.S. Dep. of Commer., Washington, D.C., 1989.

Liebe, H., P. Rosenkranz, and G. Hufford, Atmospheric $60 \mathrm{GHz}$ oxygen spectrum: New laboratory measurements and line parameter, J. Quant. Spectrosc. Radiat. Transfer, 48, 629-643, 1992.

Mizushima, M., The theory of rotating diatomic molecules, John Wiley and Sons, New York, 1975.

Palmer, P., J. Barnett, J. Eyre, and S. Healy, A non-linear optimal estimation inverse method for radio occultation measurements of temperature, humidity, and surface pressure, J. Geophys. Res., 105, 17,513-17,526, 2000. 
Pickett, H., and R. Poynter, Submillimeter, millimeter, and microwave spectral line catalogue, Applied Optics, 24, 2235-2240, 1985.

R“ais"anen, A., T. Hirvonen, J. Tuovinen, J. Ala-Laurinaho, and A. Lehto, Development of a test method for the ODIN satellite $1.1 \mathrm{~m}$ antenna at 119 and $500 \mathrm{GHz}$, in 4th International Conference on Millimeter Wave and Far Infrared Science and Technology (ICMWFST'96), pp. 13-20, Beijing, 1996.

Reburn, W., et al., Study on upper troposphere / lower stratosphere sounding, Tech. rep., ESA-ESTEC / Contract No 12053 / 97 / NL / CN, 1999.

Reburn, W. J., R. Siddans, B. J. Kerridge, S. B üler, A. von Engeln, P. Eriksson, T. Kuhn-Sander, C. Verdes, and K. Küunzi, Critical assessments in millimetre-wave atmospheric limb sounding, fi nal report, Tech. rep., ESA-ESTEC / Contract No 13348 / 98 / NL / GD, 2000.

Rodgers, C. D., Inverse Methods for Atmospheric Sounding: Theory and Practise, vol. 2 of Series on Atmospheric, Oceanic and Planetary Physics, World Scientifi c Publ., 2000.

Rosenkranz, P., Interference coeffi cients for overlapping oxygen lines in air, J. Quant. Spectrosc. Radiat. Transfer, 39, 287-297, 1988.

Rosenkranz, P., Absorption of microwaves by atmospheric gases, in Atmospheric Remote Sensing by Microwave Radiometry, edited by M. Janssen, chap. 2, pp. 37-90, John Wiley, New York, 1993.

Rothman, L., et al., The HITRAN molecular database: Editions of 1991 and 1992, J. Quant. Spectrosc. Radiat. Transfer, 48, 469507, 1992.

van Vleck, J., The absorption of microwaves by oxygen, Physical Review, 71, 413-424, 1947.
Verdes, C., S. B ühler, A. von Engeln, P. Eriksson, T. Kuhn, and K. K unzi, The retrieval of data from sub-millimetre limb sounding CNN 2, Part 1: Retrieval of pointing and temperature from non-uniformly mixed gases, Tech. rep., ESA-ESTEC / Contract No 11979 / 97 / NL / CN, 2000.

von Engeln, A., S. Büuhler, J. Langen, T. Wehr, and K. Küunzi, Retrieval of upper stratospheric and mesospheric temperature profi les from Millimeter-Wave Atmospheric Sounder data, J. Geophys. Res., 103, 31,735-31,748, 1998.

Waters, J., An overview of the EOS MLS experiment, Tech. Rep. JPL D-15745, Jet Propulsion Laboratory, Pasadena, California, 1999.

Wehr, T., S. Bühler, A. von Engeln, K. Küunzi, and J. Langen, Retrieval of stratospheric temperatures from space borne microwave limb sounding measurements, J. Geophys. Res., 103, 25,997-26,007, 1998.

S. B uhler, University of Bremen, Institute of Environmental Physics, Kufsteiner Strasse, D-28359 Bremen, Germany. (e-mail : sbuehler@uni-bremen.de; engeln@uni-bremen.de)

A. von Engeln, Naval Research Laboratory, Marine Meteorology Division, 7 Grace Hopper Avenue STOP 2, Monterey CA 93943, USA (e-mail: engeln@uni-bremen.de) Received July 5, 2001; accepted October 27, 2001.

This preprint was prepared with AGU's $\mathrm{AT}_{\mathrm{E}} \mathrm{X}$ macros v5.01, with the extension package 'AGU'+' by P. W. Daly, version 1.6b from 1999/08/19. 